endoscopically. 1 patient had surgery as the ESD specimen showed a synovial sarcoma. Endoscopic cure rate was 95.2\%.

Conclusion ESD is a safe and novel, minimal access therapeutic technique which has the potential to transform management of submucosal tumours. Patients go from the uncertainty of having repeated endoscopies for an unknown diagnosis, to having it completely removed and cured in the vast majority, without the need for continuing endoscopies. In the remaining cases, ESD specimens provide an accurate histological diagnosis based on which definite management plans can be made.

Disclosure of Interest None Declared.

\section{OC-009 A MULTICENTRE RETROSPECTIVE COMPARISON OF PLASTIC, UNCOVERED AND FULLY COVERED METAL STENTS IN THE MANAGEMENT OF DISTAL MALIGNANT BILIARY STRICTURES}

${ }^{1}$ JE Elias*, ${ }^{1} \mathrm{~F}$ Sampaziotis, ${ }^{1}$ WJ Griffiths, ${ }^{2} \mathrm{AL}$ King, ${ }^{2} \mathrm{~B}$ Macfarlane, ${ }^{1} \mathrm{JM}$ Woodward, ${ }^{1} \mathrm{GD}$ Corbett, ${ }^{2} \mathrm{~A}$ Leahy. 'Gastroenterology, Addenbrooke's Hospital, Cambridge, UK; ${ }^{2}$ Gastroenterology, Watford General Hospital, Watford, UK

10.1136/gutjnl-2014-307263.9

Introduction Uncovered self-expanding metal stents (USEMS) remain patent longer than plastic stents (PS) in patients with malignant bile duct strictures (MBDS). However, their difficult removal can compromise surgical tumour resection and so, prior to tumour staging a PS is commonly used. A solution may be offered by metal stents which have been coated with plastic, making them easier to remove. Our preliminary data analysis ${ }^{[1]}$ suggested that fully covered self-expanding metal stents (FCSEMS) used as first line management of MBDS result in a longer patency time and similar complication rates compared to USEMS and PS. We now present data from an expanded database of patients to provide further evidence of the efficacy of FCSEMS in this situation.

Methods A multicentre retrospective study was conducted of patients with MBDS who underwent ERCP and primary stenting with PS, USEMS or FCSEMS between 2007 and 2013. Data was collected from patient records on age, cancer type, stent patency time, complications and survival. Patency time was calculated as the period between stent insertion and occlusion, death or resection with a patent stent. Patients who underwent resection were excluded from the patient survival analysis. Statistical analysis used Kaplan Meier and Log Rank tests for patency and survival and Fisher's exact test for complications. The software used was IBM SPSS Statistics 20.

Results 268 patients were included. FCSEMS $(\mathrm{n}=41)$ remained patent for a mean of 292 days versus 150 days for USEMS $(\mathrm{n}=89)(\mathrm{p}<0.001)$ and 68 days for PS $(\mathrm{n}=138)$ $(\mathrm{p}<0.001)$. FCSEMS also resulted in a statistically significant improvement in patient survival with a mean of 297 days versus 191 days for USEMS ( $p<0.001$ ) and 216 days for PS ( $p$ $=0.001)$. Both FCSEMS and USEMS produced a significantly lower incidence of cholangitis than PS $(p<0.047$ and $<0.013$ respectively). There were 2 episodes of pancreatitis in the FCSEMS group (4.9\%) compared to 3 in the PS group $(2.2 \%)$ and 1 in the USEMS group (1.1\%) but this was not statistically significant.

Conclusion For primary stenting of MBDS, FCSEMS result in a longer patency time and a reduced incidence of cholangitis compared to PS. Combined with evidence supporting their ease of operative removal, this data suggests FCSEMS may be the superior option for primary stenting of MBDS. However, a larger cohort will be required to clarify the significance of the increased risk of pancreatitis observed in the FCSEMS group.

\section{REFERENCE}

1 Sampaziotis et al. A retrospective comparison of plastic, uncovered and fully covered metal stents in the management of distal malignant biliary strictures. BSG Abstract 13-1209

Disclosure of Interest None Declared.

\section{OC-010 ENDOSCOPIC RESECTION OF DUODENAL ADENOMAS - COMPARISON OF SAFETY AND EFFICACY BETWEEN SPORADIC ADENOMAS AND ADENOMAS IN FAP}

P Mundre*, L Smith, A Rehman, B Rembacken. Gastroenterology, Leeds Institute of Gastroenterology, St James's University Hospital, Leeds, Leeds, UK

\subsection{6/gutjnl-2014-307263.10}

Introduction Although there is a low risk of malignant conversion of duodenal polyps in FAP, EMR is often considered. However, few studies have looked at the safety and efficacy of EMR. We compared the outcome of duodenal EMR's in patients with FAP vs sporadic adenomas. To our knowledge, this is the largest series of duodenal EMRs that is published so far. ${ }^{1}$

Methods We looked at Clinical records of all patients who underwent endoscopic resections for duodenal adenomas at Leeds in a 10 year period.

Results A total of 49 sporadic adenomas were resected (in 51 patients) and 44 FAP related (in 22 patients). Most lesions appeared either sessile (43) or flat elevated (48). The average size of the FAP related polyps was 16.9 vs. $20.7 \mathrm{~mm}$ in sporadic lesions. Most were removed by standard EMR $(\mathrm{n}=82)$ rather than the strip biopsy technique $(n=9)$. Two procedures failed and no follow-up data was available after the resection of 2 sporadic polyps.

The final histology of the lesions were; TA+LGD (76), TA +HGD (13), adenocarcinoma (2) and 2 polyps were not retrieved. In 11 lesions, there was a change in the histological grade after resection.

There were 4 perforations (4.3\%), 3 were managed surgically. 12 patients (13\%) were readmitted with significant late GI bleeding and 8 patients required endoscopic therapy and transfusion.

There was no significant difference in the success rates in the two groups (19/44 vs. 32/49) p value 0.94). However, the resection of polyps $\geq 2 \mathrm{~cm}$ were significantly more likely to be associated with a complication $(7 / 59$ vs. $8 / 19 \mathrm{p}=0.02)$. There was no difference in the risk of complications with the polyp location, ASA status, Spigelman score or patient age.

Amongst the FAP polyps, polyps $>20 \mathrm{~mm}$ were significantly more likely to have local recurrence ( $3 / 6$ vs $3 / 31$ p value 0.04 ). There was no difference in the chances of success of the resection with the growth pattern, the location of the polyp or the Spigelman score.

Conclusion Duodenal EMR is hazardous, particularly when lesions $2 \mathrm{~cm}$ or larger are resected. However, there was no significant difference in the hazards or success rates between the two groups. Most FAP patients had further neoplasia on follow up, but this is due to the fact that many of the adenomas were not resected /treated in the first sitting.

\section{REFERENCE}

1 Basford P, Bhandari P. Endoscopic management of nonampullary duodenal polyps. Therap Adv Gastroenterol. 2012 March; 5(2):127-138

Disclosure of Interest None Declared. 